\title{
Strain-induced dispersive Landau levels: Application in twisted honeycomb magnets
}

\author{
Tianyu Liu $\odot^{1}$ and Zheng Shi $\oplus^{2}$ \\ ${ }^{1}$ Max-Planck-Institut für Physik Komplexer Systeme, 01187 Dresden, Germany \\ ${ }^{2}$ Dahlem Center for Complex Quantum Systems and Department of Physics, Freie Universität Berlin, 14195 Berlin, Germany
}

(Received 3 April 2020; revised 22 February 2021; accepted 29 March 2021; published 12 April 2021)

\begin{abstract}
Elastic strain is known to spatially modulate the wave-function overlap of the atoms on the lattice and can drastically alter the properties of the quasiparticles. For example, strain in Dirac matter can be interpreted as an elastic gauge field inducing Landau levels. We here propose a general method resolving the dispersion of the strain-induced Landau levels in two-dimensional Dirac materials, regardless of the particular space dependence of the applied strain. We illustrate such a method with the twist-induced magnon Landau levels in honeycomb quantum magnet nanoribbons. For ferromagnetic nanoribbons, dispersive Dirac-Landau levels are induced in the center of the magnon bands, while for antiferromagnetic nanoribbons, the twist results in dispersive equidistant Landau levels at the top of the magnon bands.
\end{abstract}

DOI: 10.1103/PhysRevB.103.144420

\section{INTRODUCTION}

Strain engineering is a powerful tool in tuning properties of quantum matter, such as spin transport [1,2], thermal conductivity [3,4], and quantum anomaly [5]. In particular, twisting one layer of bilayer graphene with respect to the other by certain "magic" angles [6] results in spatial modulation of interlayer electron tunneling and produces flat "Moiré bands," responsible for correlated insulators [7] and unconventional superconductors [8]. Properly tuned strain can close or open band gaps in topological quantum matter and induce phase transitions between distinct topological phases [9-14].

Perhaps the most investigated and best understood strain effects are those associated with the Dirac matter, where strain is famously equivalent to an elastic gauge field [15-33]. A circular bend in Dirac/Weyl semimetals and superconductors induces a uniform pseudomagnetic field giving rise to Landau quantization [24-26]. A uniform elastic gauge field can also occur in twisted three-dimensional Dirac nanowires $[20,28,29,32]$. Though first theoretically proposed [15] and experimentally implemented [16] in graphene, the elastic gauge field in graphene and other two-dimensional Dirac materials resulting from experimentally available simple strain such as bending [34-36] or twisting [37] is not uniform, causing difficulty in acquiring the band structure of the straininduced Landau levels (LLs).

In this paper we propose a general method in the framework of band theory to obtain the dispersion of LLs induced by a strain of arbitrary space dependence in two-dimensional

Published by the American Physical Society under the terms of the Creative Commons Attribution 4.0 International license. Further distribution of this work must maintain attribution to the author(s) and the published article's title, journal citation, and DOI. Open access publication funded by the Max Planck Society.
Dirac materials. We demonstrate that the effect of such a strain is to produce a spatially inhomogeneous elastic gauge field relocating the Dirac cones. Based on this observation, we construct a correspondence between the crystal momentum and the strain-induced elastic gauge field to derive the LL dispersion. We illustrate our method by analyzing the magnon bands of honeycomb quantum magnet nanoribbons under an experimentally available twist lattice deformation and find the dispersions of the strain-induced Dirac-Landau levels (DLLs) and equidistant Landau levels (eLLs) in ferromagnetic (FM) and antiferromagnetic (AF) nanoribbons, respectively.

The remainder of this paper is structured as follows. Our general method of finding the LL dispersion is explained in Sec. II and applied to the magnon LLs in FM and AF honeycomb nanoribbons in Secs. III and IV. Section V concludes the paper with a brief discussion. Appendix A studies the LL dispersion arising from the Aharonov-Casher effect and compares the result to the case of strains. In Appendix B we quantitatively investigate the effect of an exponentially decaying nearest-neighbor Heisenberg interaction, relaxing the simplifying assumption of a quadratic decay in Secs. III and IV. Finally, Appendix C details the recipe to find the AF magnon spectrum.

\section{GENERAL METHOD}

We first introduce our general method by considering a two-dimensional Dirac material in an elastic gauge field $\boldsymbol{B}_{\eta}=$ $\nabla \times \boldsymbol{A}_{\eta}$ varying slowly on the lattice scale, where the index $\eta$ labels the Dirac points. The vector potential $\boldsymbol{A}_{\eta}$ is incorporated into the quasiparticle Dirac Hamiltonian through the minimal substitution

$$
h_{\eta}=\sum_{j=x, y} v_{j}^{\eta}\left(\hbar q_{j}-e A_{\eta}^{j}\right) s^{j},
$$

where $s^{x, y}$ are Pauli matrices and $v_{x, y}^{\eta}$ are the velocity parameters. For a unitarily diagonalizable $h_{\eta}$, the effect of a spatially 
uniform $\boldsymbol{A}_{\eta}$ is simply relocating the $\eta$ th Dirac point from $\boldsymbol{q}=0$ to $\boldsymbol{q}=\frac{e}{\hbar} \boldsymbol{A}_{\eta}$. When $\boldsymbol{A}_{\eta}$ depends on spatial coordinates, the translational symmetry in one or both directions is broken, complicating the spectrum of $h_{\eta}$. For simplicity we assume an out-of-plane gauge field $\boldsymbol{B}_{\eta}=B_{\eta}(y) \hat{z}$, deriving from the vector potential $\boldsymbol{A}_{\eta}=A_{\eta}(y) \hat{x}$, which preserves the translational symmetry in the $x$ direction. For typical strain patterns in the experiment, the gauge field $B_{\eta}$ is nonuniform; as we show below, this gives rise to dispersive DLLs in the energy bands when transformed to the reciprocal space.

Consider the gauge field at position $y_{0}$, which displaces the Dirac point projected on the $x$ axis by $q_{x}=\frac{e}{\hbar} A_{\eta}\left(y_{0}\right)$ and thus is responsible for the band structure at $q_{x}$. Rewriting Eq. (1) in the $y$ basis,

$$
h_{\eta}(y)=\hbar v_{x}^{\eta} s^{x}\left[q_{x}-\frac{e}{\hbar} A_{\eta}(y)\right]-i \hbar v_{y}^{\eta} s^{y} \frac{d}{d y},
$$

we observe that generally the expression inside the square brackets changes sign across $y=y_{0}$. This implies the presence of a zero energy solution at $y=y_{0}$. In fact, expanding Eq. (2) in the vicinity of $y_{0}$,

$$
h_{\eta}(y) \approx \hbar v_{x}^{\eta} s^{x}\left[-\left.\frac{e}{\hbar} \frac{d A_{\eta}}{d y}\right|_{y_{0}}\left(y-y_{0}\right)\right]-i \hbar v_{y}^{\eta} s^{y} \frac{d}{d y},
$$

one immediately recognizes a minimally coupled Dirac problem. Its solutions are the familiar DLLs,

$$
E_{n}^{\eta}\left(q_{x}\right)=\operatorname{sgn}(n) \sqrt{2\left|n e \hbar v_{x}^{\eta} v_{y}^{\eta}\left(\frac{d A_{\eta}}{d y}\right)_{y_{0}}\right|} .
$$

In particular, the zeroth DLL reduces to the zero mode of Eq. (2) and traces the movement of the displaced Dirac point when $y_{0}$ is varied in the $y$ direction.

Equation (4) is the key result of this work, resolving the DLLs arising from an elastic gauge field of arbitrary space dependence. It also applies if the elastic gauge field is replaced by an ordinary electromagnetic field. The momentum dependence is incorporated by $y_{0}=A_{\eta}^{-1}\left(\frac{\hbar q_{x}}{e}\right)$, where $A_{\eta}^{-1}$ is the inverse function of the vector potential $A_{\eta}$. In general, $A_{\eta}^{-1}$ is multivalued, giving rise to a degeneracy of the strain-induced LLs.

We have retained only the term linear in $y-y_{0}$ in the expansion leading to Eq. (3), neglecting the nonuniformity of the gauge field such that Eq. (4) depends only on the local field strength at $y_{0}$. Such an adiabatic approximation is expected to work when the gauge field varies slow enough spatially to be approximated as a constant where the wave functions have support. To be more concrete, we extract the characteristic length scale of the (first few) DLL wave functions from the linearized Hamiltonian:

$$
\ell\left(y_{0}\right)=\sqrt{\left|\frac{v_{y}^{\eta} \hbar}{v_{x}^{\eta} e B_{\eta}\left(y_{0}\right)}\right|} .
$$

For the adiabatic approximation to be valid, we should have the condition

$$
\ell\left(y_{0}\right)\left|\frac{d B_{\eta}}{d y}\right|_{y_{0}} \ll\left|B_{\eta}\left(y_{0}\right)\right|
$$

We should also mention that if $A_{\eta}$ develops a large value somewhere in the system, such that the strain-induced displacement of the Dirac points at that place is comparable to the dimensions of the Brillouin zone (BZ), the effective Dirac theory in Eq. (4) becomes insufficient. This is because the velocity parameters $v_{x, y}^{\eta}$ can also be modified by the strain giving rise to $A_{\eta}[38,39]$. In such a case, a full lattice model, which treats the strain effects in elastic gauge fields and velocity parameters on equal footing, is needed as the starting point to derive a continuum theory [40].

\section{HONEYCOMB FERROMAGNETS}

In the remainder of this paper we illustrate our general method with twisted honeycomb quantum magnets characterized by the spin- $S$ Heisenberg Hamiltonian,

$$
H=\sum_{\boldsymbol{r}} \sum_{i=1}^{3} J_{i} \boldsymbol{S}_{A}(\boldsymbol{r}) \cdot \boldsymbol{S}_{B}\left(\boldsymbol{r}+\boldsymbol{\alpha}_{i}\right),
$$

where $\boldsymbol{r}$ denotes the position of an $A$ lattice site, and $\quad\left(\boldsymbol{\alpha}_{1}, \boldsymbol{\alpha}_{2}, \boldsymbol{\alpha}_{3}\right)=\left(\frac{\sqrt{3}}{2} a \hat{x}+\frac{1}{2} a \hat{y},-\frac{\sqrt{3}}{2} a \hat{x}+\frac{1}{2} a \hat{y},-a \hat{y}\right) \quad$ are nearest-neighbor vectors with $a$ being the lattice constant. We assume isotropic nearest-neighbor interaction $J_{i}=J$ in the absence of strain such that the honeycomb magnet exhibits FM (AF) order when $J<0(J>0)$ at sufficiently low temperatures. We concentrate on the FM case in this section, and discuss the AF case in Sec. IV.

An elastic strain relocates the lattice sites, resulting in the spatial modulation of wave-function overlap [41] and thus the deviation of the nearest-neighbor interactions from their strain-free value $J_{i}=J$. As a common practice, we assume that the nearest-neighbor interactions are exponentially decaying functions of distance,

$$
J_{i}=J \exp \left(-\beta \frac{\delta_{i}-\alpha_{i}}{\alpha_{i}}\right),
$$

where $\delta_{i}$ is the distance to the $i$ th nearest neighbor, and $\beta \sim 1$ is the Grüneisen parameter; the strain changes $J_{i}$ through $\delta_{i}$ alone, and effects of the orientation of anisotropic orbitals are neglected [31,42].

Our model is defined on an $L \times W$ nanoribbon twisted by an angle $\Omega$, as illustrated in Fig. 1(a). The twist deformation relocates the lattice site originally located at position $r=$ $(x, y, 0)$ to $\boldsymbol{r}+\boldsymbol{u}(\boldsymbol{r})=(x, y \cos \lambda x, y \sin \lambda x)$, where $\lambda=\Omega / L$ measures the rotational angle of a nanoribbon unit cell per unit length along the $x$ direction. The resultant bond length is then

$$
\delta_{i}=\sqrt{\alpha_{i}^{2}+\lambda^{2} \alpha_{i, x}^{2}\left(y^{2}+y \alpha_{i, y}\right)},
$$

giving exponentially decaying interactions

$$
J_{1}=J_{2}=J \exp \left[1-\sqrt{1+\frac{3}{4} \lambda^{2}\left(y^{2}+\frac{a}{2} y\right)}\right], \quad J_{3}=J .
$$

For the sake of simplicity, sometimes we shall further assume a narrow nanoribbon with a sufficiently small twist, which reduces the nearest-neighbor interactions to

$$
J_{1}=J_{2}=J-\frac{3}{8} \lambda^{2}\left(y^{2}+\frac{a}{2} y\right) J, \quad J_{3}=J .
$$


(a)

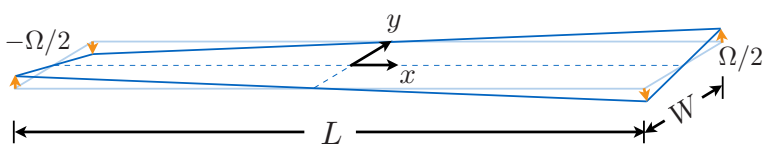

(b)

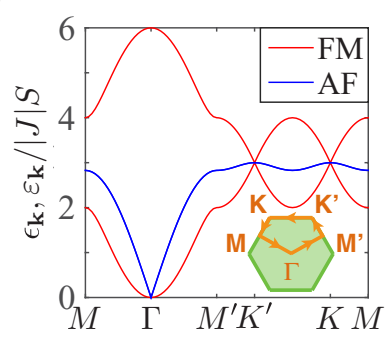

(c)

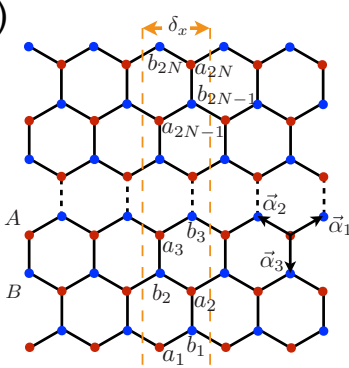

FIG. 1. (a) A twisted honeycomb magnet nanoribbon (blue) is obtained by applying torsional strain to a strain-free nanoribbon (light blue) of length $L$ and width $W$ to rotate the right (left) edge around the $x$ axis by a small angle $\Omega / 2(-\Omega / 2)$. (b) The dispersion of the honeycomb ferromagnet (red) and antiferromagnet (blue) along the path connecting the high-symmetry points of the BZ (Inset) (c) A zigzag nanoribbon with $2 N A$ and $B$ sublattice sites in a unit cell marked by two orange dashed lines. The width of the unit cell is $\delta_{x}=\sqrt{3} a$.

In the presence of FM order $(J<0)$, the Heisenberg Hamiltonian [Eq. (7)] can be second-quantized by the Holstein-Primakoff transformation [43] $S_{A}^{+}(\boldsymbol{r})=(2 S-$ $\left.a_{r}^{\dagger} a_{r}\right)^{1 / 2} a_{r}$ and $S_{A}^{z}(\boldsymbol{r})=S-a_{r}^{\dagger} a_{r}\left[S_{B}^{+}(\boldsymbol{r})=\left(2 S-b_{r}^{\dagger} b_{r}\right)^{1 / 2} b_{\boldsymbol{r}}\right.$ and $S_{B}^{z}(\boldsymbol{r})=S-b_{r}^{\dagger} b_{\boldsymbol{r}}$, where $a_{\boldsymbol{r}}\left(b_{\boldsymbol{r}}\right)$ is the magnon annihilation operator associated with the $A(B)$ sublattice. The resultant magnon tight-binding Hamiltonian to the bilinear order reads

$$
H^{\mathrm{FM}}=\sum_{\boldsymbol{r}, i} J_{i} S\left(a_{\boldsymbol{r}}^{\dagger} b_{\boldsymbol{r}+\boldsymbol{\alpha}_{i}}+a_{\boldsymbol{r}} b_{\boldsymbol{r}+\boldsymbol{\alpha}_{i}}^{\dagger}-a_{\boldsymbol{r}}^{\dagger} a_{\boldsymbol{r}}-b_{\boldsymbol{r}}^{\dagger} b_{\boldsymbol{r}}\right),
$$

where the FM ground-state energy $E_{G}^{\mathrm{FM}}=\sum_{r, i} J_{i} S^{2}$ has been subtracted from the Heisenberg Hamiltonian. For isotropic and spatially uniform $J_{i}=J$, the Fourier transform $\left(a_{\boldsymbol{r}}, b_{\boldsymbol{r}}\right)^{T}=N_{\mathrm{uc}}^{-1 / 2} \sum_{\boldsymbol{k}} e^{i k \cdot \boldsymbol{r}}\left(a_{\boldsymbol{k}}, b_{\boldsymbol{k}}\right)^{T}$, where $N_{\mathrm{uc}}$ is the number of unit cells, maps Eq. (12) to

$$
\mathcal{H}_{\boldsymbol{k}}=\sum_{i} J_{i} S\left[\cos \left(\boldsymbol{k} \cdot \boldsymbol{\alpha}_{i}\right) \sigma^{x}-\sin \left(\boldsymbol{k} \cdot \boldsymbol{\alpha}_{i}\right) \sigma^{y}-\sigma^{0}\right],
$$

where Pauli matrices $\sigma^{x, y}$ and the identity matrix $\sigma^{0}$ are defined in the sublattice basis $\left(a_{k}, b_{k}\right)^{T}$. The dispersion of this Bloch Hamiltonian reads

$$
\epsilon_{k}=3|J S| \pm\left|J S \sum_{i} e^{i k \cdot \alpha_{i}}\right|,
$$

which exhibits two Dirac cones [Fig. 1(b)] at the BZ corners $\boldsymbol{k}_{W}^{\eta}=\left(\eta \frac{4 \pi}{3 \sqrt{3} a}, 0\right)$ with $\eta= \pm 1$. For a nanoribbon with a pair of zigzag edges along the $x$ direction [Fig. 1(c)], the band $\epsilon_{\boldsymbol{k}}$ becomes a cluster of bands [Fig. 2(a)].

We introduce a fictitious lattice deformation that alters the nearest-neighbor interactions according to

$$
J_{1}=J_{2}=J+\delta J, \quad J_{3}=J,
$$

(a)

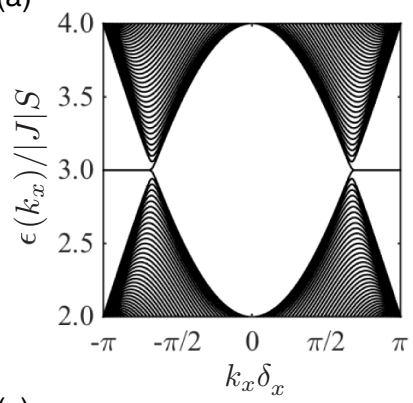

(c)

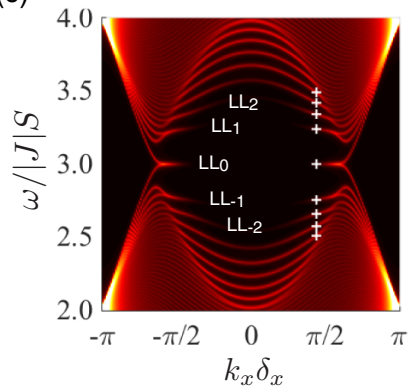

(b)

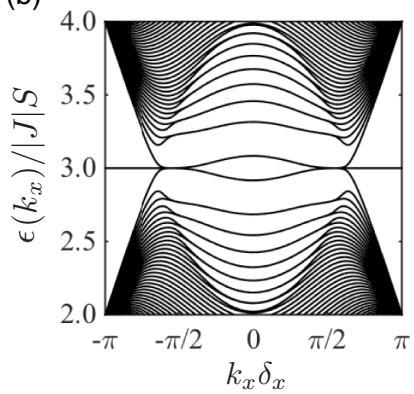

(d)

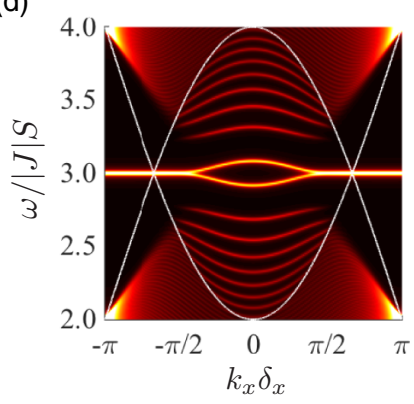

FIG. 2. Spectral properties of a zigzag FM nanoribbon with $2 N=80 A$ and $B$ sublattice sites. (a) The spectrum of an untwisted nanoribbon exhibiting two Dirac cones connected by flat edge states. (b) The spectrum of a twisted nanoribbon with $\lambda a=0.032$. (c) The bulk spectral function of (b) with dispersive DLLs in the vicinity of $K$ valley labeled. The crosses mark the positions of DLLs at the selected momentum $k_{x} \delta_{x}=1.382$. (d) The edge spectral function of (b) with the white curves representing the envelopes of the Dirac cones.

where the variation $\delta J$ is spatially uniform. In the presence of such a deformation, an effective Dirac theory can be obtained by linearizing the Bloch Hamiltonian [Eq. (13)] in the vicinity of BZ corners:

$$
h_{q}=\hbar v_{x}^{\eta}\left(q_{x}+\eta \frac{2 \delta J}{3 a J}\right) \sigma^{x}+\hbar v_{y}^{\eta} q_{y} \sigma^{y}-(3 J S+2 \delta J S) \sigma^{0},
$$

where the magnon velocity is $\left(v_{x}^{\eta}, v_{y}^{\eta}\right)=\frac{3 J S a}{2 \hbar}(-\eta, 1)$. We note that $\delta J$ has two effects. On the one hand, it shifts the two Dirac cones uniformly in the energy dimension by an amount of $-2 \delta J S$. This effect is rather trivial and can be greatly suppressed by a Zeeman field $B_{Z}=-2 \delta J S / g \mu_{B}$, where $g \mu_{B}$ is the magnon magnetic moment. Therefore we will neglect this effect in the following. On the other hand, $\delta J$ displaces the two Dirac cones oppositely in the momentum dimension by $\eta \frac{2 \delta J}{3 a J}$. Although an electric field can also relocate the magnon Dirac cones through the Aharonov-Casher (AC) effect [44] (see Appendix A), the two Dirac cones are always translated identically, implying that the valley-sensitive displacement should be interpreted as an axial elastic vector potential

$$
\overrightarrow{\mathcal{A}}=\eta \frac{2 \hbar}{3 e a} \frac{\delta J}{J} \hat{x}
$$

which cannot be compensated by electric fields.

We have obtained $\overrightarrow{\mathcal{A}}$ using a uniform $\delta J$. However, we are interested in a twist deformation [Fig. 1(a)], where $\delta J$ and the resulting $\overrightarrow{\mathcal{A}}$ depend on the $y$ coordinate. According 

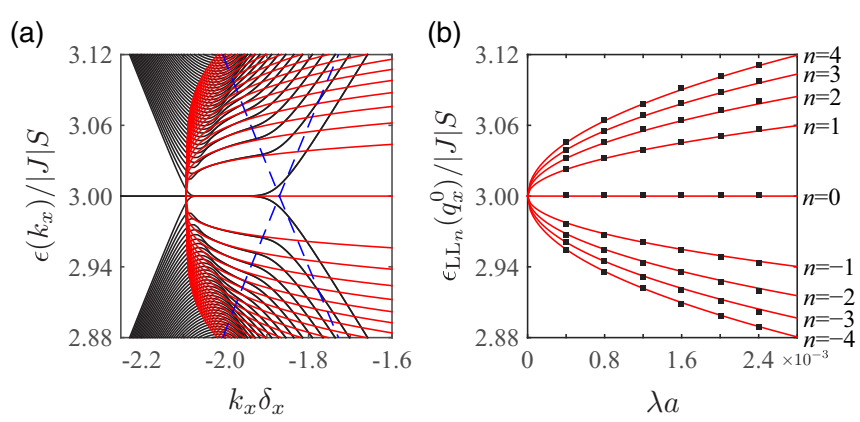

FIG. 3. Dispersive DLLs in a twisted FM nanoribbon. (a) Numerically calculated magnon bands (black) for a nanoribbon with $2 N=1200 A$ and $B$ sublattice sites and $\lambda a=0.0008$. The theoretically predicted DLLs (red) from Eq. (19) are overlaid. The dashed blue curves mark the position of the maximally displaced Dirac cone, which is $q_{x}^{w} \delta_{x}=0.225$ on the right of valley $K$. (b) DLLs as a function of twist at a fixed momentum that is $q_{x}^{0} \delta_{x}=0.139$ on the right of the $K$ point. The theoretically predicted DLLs (red) from Eq. (19) well fit the numerics (black dots) of the first few DLLs.

to the general method we have developed, this nonuniform $\overrightarrow{\mathcal{A}}$ should induce dispersive DLLs. To substantiate our claim, we numerically simulate the tight-binding Hamiltonian of a twisted zigzag nanoribbon with exponentially decaying interactions [Eq. (10)]. Indeed, dispersive DLLs are induced on right (left) of valley $K\left(K^{\prime}\right)$ [Fig. 2(b)], reflecting the valley sensitivity of $\overrightarrow{\mathcal{A}}$. These DLLs are doubly degenerate due to the contributions from the upper $(y>0)$ and lower $(y<0)$ sections of the nanoribbon. To better resolve these DLLs, we calculate the spectral function

$$
A\left(\omega, k_{x}\right)=-\frac{1}{\pi} \sum_{y} \lim _{\delta \rightarrow 0} \Im\left[\omega+i \delta-\mathcal{H}_{k_{x}}\right]_{y y}^{-1},
$$

in the bulk and on the edges of the nanoribbon. The bulk origin of these DLLs is confirmed by the bulk spectral function, defined to include the contribution of the central $50 \%$ lattice sites. Extracting the energies $\epsilon_{\mathrm{LL}_{n}}$ of the first few DLLs marked by the crosses in Fig. 2(c), we find the sequence $\epsilon_{\mathrm{LL}_{n}}-\epsilon_{\mathrm{LL}_{0}}$ exhibits the expected $\sqrt{n}$ dependence on the LL index $n$. We note that the dispersive DLLs only reside in the vicinity of Dirac cones. This observation is best demonstrated by the fact that the first three DLLs $(n=0, \pm 1, \pm 2)$ associated with each valley are not connected through the bulk but by the edge states, as illustrated by the edge spectral function [Fig. 2(d)].

We now analyze the twist-induced DLLs in a more quantitative way. Here we assume a sufficiently small twist under which Eq. (11) is applicable; a more generic derivation regarding large twists is given in Appendix B. Following our general method, we write the axial vector potential as a function of coordinate, $\mathcal{A}_{x}(y)=-(\eta \hbar / 4 e a) \lambda^{2} y^{2}$. The resulting twistinduced DLLs read

$$
\epsilon_{\mathrm{LL}_{n}}\left(q_{x}\right)=-3 J S-\frac{3}{2} J S \sqrt{\lambda a} \sqrt[4]{4 a q_{x}} \operatorname{sgn}(n) \sqrt{|n|} .
$$

Numerical simulation confirms that Eq. (19) well captures the dispersion of the DLLs [Fig. 3(a)]. For a fixed momentum slightly away from the Dirac point $K$, we also test the $\sqrt{\lambda}$ dependence of the first few DLLs. These results are summa- rized in Fig. 3(b). Far away from the valley $K$, the numerically obtained bands begin to deviate from the prediction of Eq. (19), as the wave functions move to the edge of the nanoribbon and evolve from bulk DLLs into edge states. The width of the zeroth DLL corresponds to the maximal displacement of the Dirac cone

$$
q_{x}^{w}=\left[-\frac{e}{\hbar} \mathcal{A}_{x}(y)\right]_{\max }=\frac{\lambda^{2} W^{2}}{16 a}
$$

as $y$ traverses the width of the nanoribbon $-\frac{W}{2} \leqslant y \leqslant \frac{W}{2}$; higher-order DLLs have smaller widths because of the larger spatial extent of their wave functions. Therefore Eq. (19) fits the numerics best between the Dirac cone at $K$ and the maximally displaced Dirac cone.

Finally, let us verify that the DLLs in Eq. (19) satisfy the adiabatic condition Eq. (6). Noting that the first DLL should be outside of the projected Dirac cones, we have

$$
-\frac{3}{2} J S \sqrt{\lambda a}\left(4 q_{x} a\right)^{\frac{1}{4}} \ll-\frac{3}{2} J S q_{x} a ;
$$

on the other hand, Eq. (6) yields

$$
q_{x} a=\frac{1}{4} \lambda^{2} y_{0}^{2} \gg \frac{1}{4}(2 \lambda a)^{\frac{2}{3}} .
$$

It is straightforward to check that Eqs. (21) and (22) agree up to an $O(1)$ constant. Therefore, for a weakly twisted FM nanoribbon, the assumption of a slowly varying gauge field is self-consistent as long as the DLLs fall outside of the Dirac cones.

\section{HONEYCOMB ANTIFERROMAGNETS}

In the presence of AF order $(J>0)$, the Heisenberg Hamiltonian [Eq. (7)] can be second-quantized by the bipartite Holstein-Primakoff transformation $S_{A}^{+}(\boldsymbol{r})=(2 S-$ $\left.a_{r}^{\dagger} a_{\boldsymbol{r}}\right)^{1 / 2} a_{\boldsymbol{r}}$ and $S_{A}^{z}=S-a_{\boldsymbol{r}}^{\dagger} a_{\boldsymbol{r}}\left[S_{B}^{+}(\boldsymbol{r})=b_{\boldsymbol{r}}^{\dagger}\left(2 S-b_{\boldsymbol{r}}^{\dagger} b_{\boldsymbol{r}}\right)^{1 / 2}\right.$ and $\left.S_{B}^{z}=b_{r}^{\dagger} b_{r}-S\right]$. The resulting magnon tight-binding Hamiltonian at the noninteracting level is

$$
H^{\mathrm{AF}}=\sum_{\boldsymbol{r}, i} J_{i} S\left(a_{\boldsymbol{r}} b_{\boldsymbol{r}+\boldsymbol{\alpha}_{i}}+a_{\boldsymbol{r}}^{\dagger} b_{\boldsymbol{r}+\boldsymbol{\alpha}_{i}}^{\dagger}+a_{\boldsymbol{r}}^{\dagger} a_{\boldsymbol{r}}+b_{\boldsymbol{r}}^{\dagger} b_{\boldsymbol{r}}\right),
$$

where the Néel state energy $E_{N}=-\sum_{r, i} J_{i} S^{2}$ has been subtracted from the Heisenberg Hamiltonian. For isotropic and spatially uniform $J_{i}=J$, the Fourier transform maps Eq. (23) to

$$
\mathscr{H}_{\boldsymbol{k}}=\sum_{i} J S\left[\cos \left(\boldsymbol{k} \cdot \boldsymbol{\alpha}_{i}\right) \tau^{x}-\sin \left(\boldsymbol{k} \cdot \boldsymbol{\alpha}_{i}\right) \tau^{y}+\tau^{0}\right],
$$

which is identical to Eq. (13), except that Pauli matrices $\tau^{x, y}$ and the identity matrix $\tau^{0}$ are defined in the particle-hole basis $\left(a_{k}, b_{-k}^{\dagger}\right)^{T}$. Therefore the AF honeycomb magnet can be regarded as a generalized Dirac material whose Hamiltonian is diagonalized by a paraunitary Bogoliubov transformation in order to maintain the bosonic statistics of the basis (see Appendix C). Explicitly, the dispersion of the AF Bloch Hamiltonian is

$$
\varepsilon_{\boldsymbol{k}}=\sqrt{(3 J S)^{2}-\left(\epsilon_{\boldsymbol{k}}-3|J S|\right)^{2}}=J S \sqrt{9-\left|\sum_{i} e^{i k \cdot \boldsymbol{\alpha}_{i}}\right|^{2}}
$$

where $\epsilon_{\boldsymbol{k}}$ is the dispersion of the FM Bloch Hamiltonian given by Eq. (14). Equation (25) exhibits two quadratic peaks at BZ 
(a)

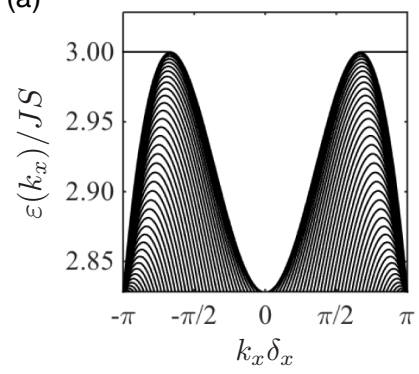

(c)

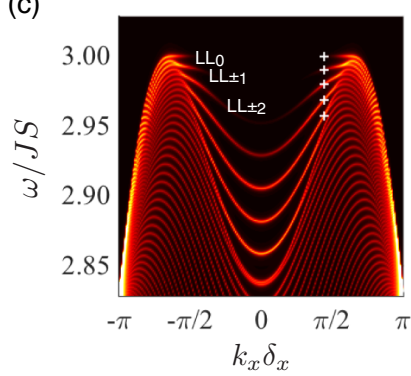

(b)

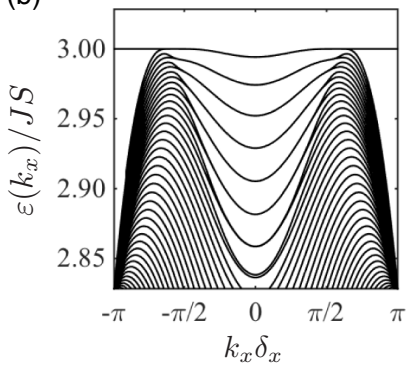

(d)

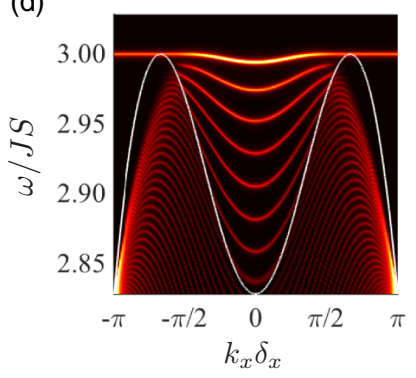

FIG. 4. Spectral properties of a zigzag AF nanoribbon with $2 N=80 A$ and $B$ sublattice sites. (a) The spectrum of an untwisted nanoribbon exhibiting two quadratic peaks connected by flat edge states. (b) The spectrum of a twisted nanoribbon with $\lambda a=0.028$. (c) The bulk spectral function of (b) with dispersive eLLs in the vicinity of quadratic peak $K$ labeled. The crosses mark the positions of eLLs at the selected momentum $k_{x} \delta_{x}=1.382$. (d) The edge spectral function of (b) with white curves representing the envelopes of the quadratic peaks.

corners $K / K^{\prime}$ [Fig. 1(b)] and becomes a set of bands for a zigzag nanoribbon [Fig. 4(a)].

We now consider the effect of the fictitious lattice deformation Eq. (15). Based on the close relation between the dispersion relations of FM and AF honeycomb magnets [Eq. (25)], we can directly write down the magnon dispersion for an AF honeycomb magnet in the vicinity of the BZ corners as

$$
\varepsilon_{\boldsymbol{q}}=(3 J S+2 \delta J S)-\frac{3 J S}{8} a^{2}\left[\left(q_{x}+\eta \frac{2 \delta J}{3 a J}\right)^{2}+q_{y}^{2}\right],
$$

where the constant $\delta J$ can still be interpreted as resulting from an axial elastic vector potential as in ferromagnets [Eq. (17)]. Therefore, a $y$-dependent $\delta J$ emerging from the twist should induce LLs in AF honeycomb magnets, similar to the $\delta J$ in FM honeycomb magnets.

To support our argument, we numerically calculate the band structure of a zigzag AF nanoribbon with exponentially decaying interactions [Eq. (10)]. We find dispersive eLLs on the right (left) of quadratic peak $K\left(K^{\prime}\right)$ [Fig. 4(b)]. These eLLs are fourfold degenerate except for the zeroth eLL, which is doubly degenerate. For the energies $\varepsilon_{\mathrm{LL}_{n}}$ of the first few eLLs marked by the crosses in Fig. 4(c), the sequence $\varepsilon_{\mathrm{LL}_{n}}-\varepsilon_{\mathrm{LL}_{0}}$ indeed shows the expected linear dependence on the LL index $n$. This is consistent with the quadratic dispersion [Eq. (26)] at the BZ corners. The bulk spectral function [Fig. 4(c)] confirms the bulk origin of the eLLs with the best resolution for the first three eLLs $(n=0, \pm 1, \pm 2)$ of each quadratic peak, which are connected by edge states rather than

(a)

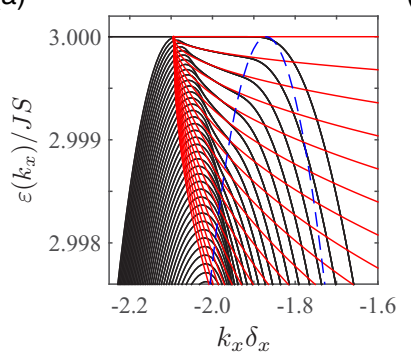

(b)

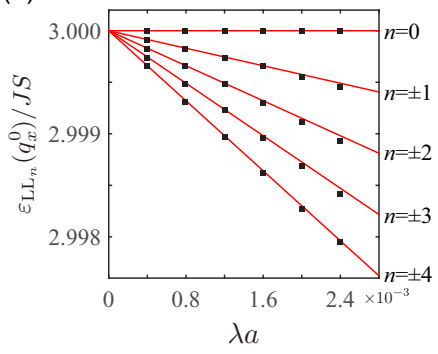

FIG. 5. Dispersive eLLs in a twisted AF nanoribbon. (a) Numerically calculated band structure (black) for a nanoribbon with $2 N=$ $1200 A$ and $B$ sublattice sites and $\lambda a=0.0008$. The theoretically predicted eLLs (red) from Eq. (27) are overlaid. The dashed blue curves mark the position of the maximally displaced quadratic peak, which is $q_{x}^{w} \delta_{x}=0.225$ on the right of quadratic peak $K$. (b) eLLs as a function of twist at fixed momentum, which is $q_{x}^{0} \delta_{x}=0.139$ on the right of the $K$ point. The theoretically predicted eLLs (red) from Eq. (27) well fit the numerics (black dots) of the first few eLLs.

through the bulk as illustrated by the edge spectral function [Fig. 4(d)].

For a sufficiently small twist, the dispersion of the induced eLLs can be immediately written down by making use of Eq. (19) as

$$
\varepsilon_{\mathrm{LL}_{n}}\left(q_{x}\right)=3 J S-\frac{3}{8} J S \lambda a \sqrt{4 a q_{x}}|n|,
$$

which is numerically verified by direct diagonalization of the tight-binding Hamiltonian of a zigzag AF nanoribbon with quadratically decaying interactions [Eq. (11)]. Indeed, we find Eq. (27) well captures the eLL dispersion between the quadratic peak at $K$ and the maximally displaced quadratic peak [Fig. 5(a)], whose displacement is again specified by Eq. (20). We also examine the linear $\lambda$ dependence of the first few eLLs at a fixed momentum slightly away from $K$. These results are summarized in Fig. 5(b). We comment in passing that, as a result of the intimate relation Eq. (25) between FM and AF dispersions, the adiabatic condition Eq. (6) is again satisfied for the eLLs in Eq. (27).

\section{CONCLUSIONS}

We have proposed a general method resolving the dispersion of Landau levels induced by strain of arbitrary space dependence in two-dimensional Dirac materials. The effectiveness of the method is verified by successfully acquiring the strain-induced magnon Landau levels in both ferromagnetic and antiferromagnetic twisted honeycomb magnet nanoribbons. Our proposal may be experimentally carried out with honeycomb ferromagnets $\mathrm{Cr} X_{3}(X=\mathrm{F}, \mathrm{Cl}, \mathrm{Br}, \mathrm{I})[45,46]$ and antiferromagnet $\mathrm{MnPS}_{3}$ [47]. The required Zeeman field canceling the twist-induced onsite energy may be provided by a fine-tuned array of magnetic force microscope tips [48], and the magnon Landau levels can be imaged by neutron scattering [49].

Although the method we proposed is only illustrated with honeycomb ferromagnets and antiferromagnets, it should be generally applicable to other two-dimensional Dirac materials, such as those containing photons [18], phonons [50,51], 
and Majorana particles [52,53], where the strain-induced Landau levels occur. In particular, in graphene [38,54], kagome metals [33], and $d$-wave superconductors [27,30], the straininduced Landau levels are known to be dispersive, making these materials an ideal venue to examine the validity of our theory. In a recent work by the present authors [40], the method reported here is further developed to account for the band structure of a twisted graphene nanoribbon in the presence of very strong strain.

\section{ACKNOWLEDGMENTS}

The authors are indebted to R. Moessner, M. Franz, and H. Kondo for insightful discussions. T.L. gratefully acknowledges the Department of Physics of SUSTech for the accommodation during the revision of the manuscript. Z.S. is supported by Project A02 of the CRC-TR 183.

\section{APPENDIX A: MAGNON LANDAU LEVELS DUE TO THE AHARONOV-CASHER EFFECT}

In Secs. III and IV, we have shown that a twist lattice deformation spatially modulates the nearest-neighbor spin interaction, thus resulting in magnon LLs in the vicinity of the BZ corners. In this section we will study the magnon LLs resulting from the $\mathrm{AC}$ effect, paying close attention to its difference from the twist lattice deformation.

We consider an isotropic Heisenberg model in the presence of an external electric field $\boldsymbol{E}$,

$$
\begin{aligned}
H= & \sum_{\boldsymbol{r}, i} J\left[e^{i \phi_{A C}} S_{A}^{-}(\boldsymbol{r}) S_{B}^{+}\left(\boldsymbol{r}+\boldsymbol{\alpha}_{i}\right)+\text { H.c. }\right] \\
& +\sum_{\boldsymbol{r}, i} J S_{A}^{z}(\boldsymbol{r}) S_{B}^{z}\left(\boldsymbol{r}+\boldsymbol{\alpha}_{i}\right),
\end{aligned}
$$

where $\phi_{A C}=\frac{1}{\hbar c^{2}} \int_{\boldsymbol{r}}^{\boldsymbol{r}+\boldsymbol{\alpha}_{i}}\left(\boldsymbol{\mu}_{s} \times \boldsymbol{E}\right) \cdot d \boldsymbol{l}$ is the AC phase, in which $\boldsymbol{\mu}_{s}=-g \mu_{B} \hat{z}$ is the magnetic moment of the spin transported from the $A$ site at $\boldsymbol{r}$ to the neighboring $B$ site at $\boldsymbol{r}+\boldsymbol{\alpha}_{i}$. By applying Holstein-Primakoff transformation and the Fourier transform, it is easy to find that the effect of the AC phase is to displace the magnon bands according to the Peierls substitution $\boldsymbol{k} \rightarrow \boldsymbol{k}+\frac{1}{\hbar c^{2}}\left(\boldsymbol{\mu}_{s} \times \boldsymbol{E}\right)$ for both ferromagnets and antiferromagnets. Therefore a spatially varying electric field $\boldsymbol{E}$ satisfying $\nabla \times\left(\boldsymbol{\mu}_{s} \times \boldsymbol{E}\right) \neq 0$ can lead to LLs at the BZ corners. However, it is crucially important to notice that there are two major differences between the $\mathrm{AC}$ effect and the twist: (i) The AC phase shifts Dirac cones (quadratic peaks) of ferromagnets (antiferromagnets) at $K$ and $K^{\prime}$ in the same direction, while the twist always displaces two different Dirac cones (quadratic peaks) oppositely. (ii) The AC phase not only shifts the magnon Dirac cones (quadratic peaks) at the BZ corners, but also relocates magnon bands of ferromagnets (antiferromagnets) at other momenta through $\boldsymbol{k} \rightarrow \boldsymbol{k}+\frac{1}{\hbar c^{2}}\left(\boldsymbol{\mu}_{s} \times \boldsymbol{E}\right)$. In contrast, a twist only impacts the magnon Dirac cones (quadratic peaks), while the magnon bands far away from the BZ corners are not significantly affected. To illustrate these differences, we perform numerical simulations for zigzag nanoribbons of both honeycomb ferromagnets and antiferromagnets. The nonuniform electric field is chosen such that $\frac{1}{e c^{2}}\left(\boldsymbol{\mu}_{s} \times \boldsymbol{E}\right)=-\frac{\hbar}{4 e a} \lambda_{\text {eff }}^{2} y^{2} \hat{x}$, which allows us to parametrize
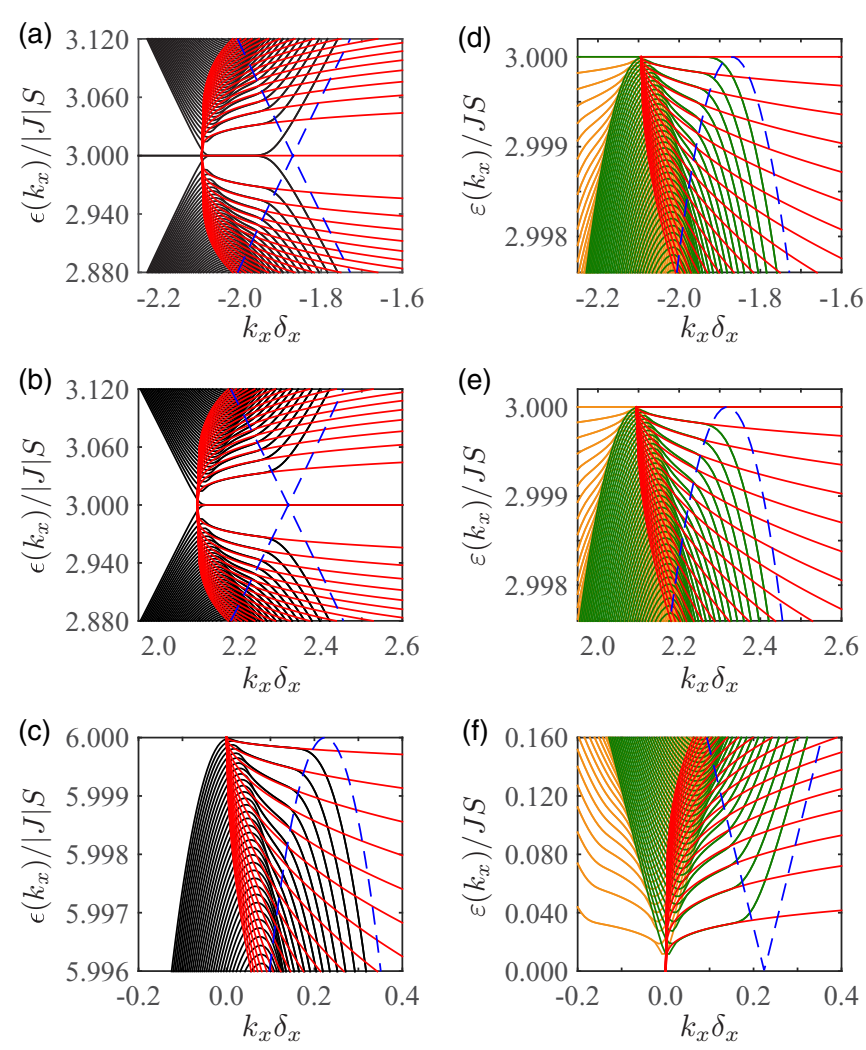

FIG. 6. Magnon band structure for nanoribbons with $2 N=1200$ $A$ and $B$ sites in the unit cell in the presence of an external electric field $\boldsymbol{E}$, which is chosen to satisfy $\frac{1}{e c^{2}}\left(\boldsymbol{\mu}_{s} \times \boldsymbol{E}\right)=-\frac{\hbar}{4 e a} \lambda_{\text {eff }}^{2} y^{2} \hat{x}$ with $\lambda_{\text {eff }} a=0.0008$. (a)-(c) Magnon LLs of ferromagnetic nanoribbons resulting from the AC effect on the right of high-symmetry points $K$, $K^{\prime}$, and $\Gamma$, respectively. The theoretically proposed LLs [Eq. (A2) for $(\mathrm{a}, \mathrm{b})$ and Eq. (A3) for (c)] are sketched as red curves and overlaid to the corresponding panels. (d)-(f) Magnon LLs of antiferromagnetic nanoribbons resulting from the AC effect in the vicinity of highsymmetry points $K, K^{\prime}$, and $\Gamma$, respectively. The green curves and the orange curves are from distinct pseudospin sectors, i.e., $\mathscr{H}_{\boldsymbol{k}}$ and $\mathscr{H}_{-\boldsymbol{k}}^{*}$ in the magnon BdG Hamiltonian [Eq. (C13)]. The theoretically proposed LLs [Eq. (A4) for (d,e) and Eq. (A5) for (f)] are sketched as red curves and overlaid to the corresponding panels. For all panels, the dashed blue curves represent the maximally displaced cones/peaks on the right of each high-symmetry point by $q_{x}^{w} \delta_{x}=0.225$, which is same as those used for Figs. 3(a) and 5(a).

the electric field by an "effective twist" $\lambda_{\text {eff. }}$ Without loss of generality, we choose $\lambda_{\text {eff }}>0$.

For ferromagnetic nanoribbons $(J<0)$, we find that the dispersive DLLs due to the AC effect appear on the right of valley $K$ [Fig. 6(a)] and valley $K^{\prime}$ [Fig. 6(b)]. The dispersion of these LLs can be immediately written down by referring to Eq. (19):

$$
\begin{aligned}
\epsilon_{\mathrm{LL}_{n}}^{K / K^{\prime}}\left(q_{x}\right) & =-3 J S+\operatorname{sgn}(n) \sqrt{\left|2 n \frac{e}{\hbar} \boldsymbol{B}_{\mathrm{eff}} \hbar v_{x}^{\eta} \hbar v_{y}^{\eta}\right|} \\
& =-3 J S-\frac{3}{2} J S \sqrt{\lambda_{\mathrm{eff}}} a \sqrt[4]{4 a q_{x}} \operatorname{sgn}(n) \sqrt{|n|},
\end{aligned}
$$

where $\left(v_{x}^{\eta}, v_{y}^{\eta}\right)=\frac{3 J S a}{2 \hbar}(-\eta, 1)$ is the magnon velocity associated with each valley, with index $\eta=+1(-1)$ representing 
valley $K\left(K^{\prime}\right)$ and the effective gauge field $\boldsymbol{B}_{\text {eff }}=\frac{1}{e c^{2}} \nabla \times$ $\left(\boldsymbol{\mu}_{s} \times \boldsymbol{E}\right)=\frac{\hbar}{2 e a} \lambda_{\text {eff }}^{2} y \hat{z}$. On the other hand, the twist-induced LLs reside on the right (left) of valley $K\left(K^{\prime}\right)$, reflecting the fundamental difference between the AC effect and the twist. Moreover, on the right of the $\Gamma$ point, the $\mathrm{AC}$ effect results in dispersive eLLs. This is because magnon dispersion in the vicinity of the $\Gamma$ point is quadratic $\epsilon_{q}^{\Gamma}=-3 J S \pm 3 J S[1-$ $\left.\frac{1}{4} a^{2}\left(q_{x}^{2}+q_{y}^{2}\right)\right]$, giving rise to the dispersion

$$
\begin{aligned}
\epsilon_{\mathrm{LL}_{n}}^{\Gamma}\left(q_{x}\right) & =-3 J S \pm\left[3 J S+\hbar \omega_{c}\left(n+\frac{1}{2}\right)\right] \\
& =-3 J S \pm 3 J S \mp \frac{3}{4} J S \lambda_{\text {eff }} a \sqrt{4 q_{x} a}\left(n+\frac{1}{2}\right),
\end{aligned}
$$

where $\omega_{c}=\left|\frac{3 J S a^{2}}{2 \hbar^{2} c^{2}} \nabla \times\left(\boldsymbol{\mu}_{s} \times \boldsymbol{E}\right)\right|$ is the magnon cyclotron frequency. Such eLLs are absent in twisted FM nanoribbons because the twist-induced gauge field only couples to the magnon Dirac cones and has little effect on the magnon bands far away from the BZ corners.

For antiferromagnetic nanoribbons $(J>0)$, we find dispersive eLLs on the right of quadratic peaks $K$ and $K^{\prime}$ [green curves in Fig. 6(d) and 6(e)]. By referring to Eq. (27), we find these LLs are characterized by

$$
\begin{aligned}
\varepsilon_{\mathrm{LL}_{n}}^{K / K^{\prime}}\left(q_{x}\right) & =\sqrt{(3 J S)^{2}-\left|2 n \frac{e \boldsymbol{B}_{\text {eff }}}{\hbar} \hbar v_{x}^{\eta} \hbar v_{y}^{\eta}\right|} \\
& =3 J S-\frac{3}{8} J S \lambda_{\text {eff }} a \sqrt{4 a q_{x}}|n| .
\end{aligned}
$$

The AC effect also produces on the right of the $\Gamma$ point additional modified DLLs [green curves in Fig. 6(f)],

$$
\begin{aligned}
\varepsilon_{\mathrm{LL}_{n}}^{\Gamma}\left(q_{x}\right) & =\sqrt{(3 J S)^{2}-\left[3 J S-\hbar \omega_{c}\left(n+\frac{1}{2}\right)\right]^{2}} \\
& =\frac{3}{\sqrt{2}} J S \sqrt{\lambda_{\text {eff }} a} \sqrt[4]{4 q_{x} a} \sqrt{n+\frac{1}{2}},
\end{aligned}
$$

because the magnon bands exhibit linear dispersion $\varepsilon_{q}^{\Gamma}=$ $\frac{3}{\sqrt{2}} J \operatorname{Sa}\left[q_{x}^{2}+q_{y}^{2}\right]^{1 / 2}$ in the vicinity of the $\Gamma$ point. It is worth noting that there are additional LLs on the left of highsymmetry points $K, K^{\prime}$, and $\Gamma$, as illustrated by the orange curves in Figs. 6(d)-6(f). The symmetric distribution of these LLs arises from the inherent symmetry of the magnon Bloch Hamiltonian $\mathscr{H}_{\boldsymbol{k}}(\boldsymbol{E})=\mathscr{H}_{-\boldsymbol{k}}^{*}(-\boldsymbol{E})$, which can be justified by noting that the Bloch phase factor (AC phase factor) in $\mathscr{H}_{\boldsymbol{k}}(\boldsymbol{E})$ is invariant under the consecutive complex conjugation and inversion $\boldsymbol{k} \rightarrow-\boldsymbol{k}(\boldsymbol{E} \rightarrow-\boldsymbol{E})$. In the presence of this symmetry, the magnon BdG Hamiltonian on the bipartite honeycomb lattice [Eq. (C13)] decouples into two sectors $\mathscr{H}_{\boldsymbol{k}}(\boldsymbol{E})$ and $\mathscr{H}_{-k}^{*}(\boldsymbol{E})=\mathscr{H}_{\boldsymbol{k}}(-\boldsymbol{E})$, which produce LLs on the right and left of high-symmetry points, respectively.

\section{APPENDIX B: DERIVATION OF LANDAU-LEVEL DISPERSION FOR LARGE TWISTS}

The LL dispersions, Eqs. (19) and (27), are obtained under the assumption that the twist is sufficiently small such that Eq. (11) is a good estimate for the nearest-neighbor interactions $J_{i}$. However, when the twist is large, it is more reasonable to assume exponentially decaying interactions as in Eq. (10). In this section we will derive the dispersion of magnon LLs for a generic twist for both honeycomb ferromagnets (Appendix B 1) and antiferromagnets (Appendix B 2).

\section{Honeycomb ferromagnets}

Starting from the fictitious lattice deformation Eq. (15), we linearize the FM Bloch Hamiltonian Eq. (13) in the vicinity of the BZ corners as

$$
\begin{aligned}
\tilde{h}_{q}= & \hbar \tilde{v}_{x}^{\eta}\left[q_{x}+\eta \frac{2 \delta J}{3 a(J+\delta J)}\right] \sigma^{x}+\hbar \tilde{v}_{y}^{\eta} q_{y} \sigma^{y} \\
& -(3 J S+2 \delta J S) \sigma^{0}
\end{aligned}
$$

where $\left(\tilde{v}_{x}^{\eta}, \tilde{v}_{y}^{\eta}\right)=\frac{3 J S a}{2 \hbar}\left(-\eta \frac{J+\delta J}{J}, \frac{3 J+\delta J}{3 J}\right)$ is the new magnon velocity. When $\delta J \ll J$, this reduces to the magnon velocity in Eq. (16). The emergent vector potential resulting from $\delta J$ can be directly read off Eq. (B1) as $\tilde{\mathcal{A}}_{x}=\eta \frac{2 \hbar}{3 a e} \frac{\delta J}{J+\delta J}$.

We have derived the magnon velocity and the emergent vector potential by assuming a constant $\delta J$. In fact, we are interested in the twist lattice deformation, where $\delta J=\delta J(y)$ is space dependent [Eq. (10)], giving rise to the modified magnon velocity

$$
\begin{aligned}
& \tilde{v}_{x}^{\eta}=-\eta \frac{3 J S a}{2 \hbar} \exp \left[1-\sqrt{1+\frac{3}{4} \lambda^{2}\left(y^{2}+\frac{a}{2} y\right)}\right], \\
& \tilde{v}_{y}^{\eta}=\frac{J S a}{2 \hbar}\left\{2+\exp \left[1-\sqrt{1+\frac{3}{4} \lambda^{2}\left(y^{2}+\frac{a}{2} y\right)}\right]\right\},
\end{aligned}
$$

and the twist-induced vector potential

$$
\tilde{\mathcal{A}}_{x}=\eta \frac{2 \hbar}{3 a e}\left\{1-\exp \left[\sqrt{1+\frac{3}{4} \lambda^{2}\left(y^{2}+\frac{a}{2} y\right)}-1\right]\right\},
$$

whose curl gives the twist-induced elastic gauge field

$$
\tilde{\mathcal{B}}_{z}=\eta \frac{\hbar}{2 a e} \lambda^{2} y \frac{\exp \left[\sqrt{1+\frac{3}{4} \lambda^{2}\left(y^{2}+\frac{a}{2} y\right)}-1\right]}{\sqrt{1+\frac{3}{4} \lambda^{2}\left(y^{2}+\frac{a}{2} y\right)}} .
$$

As we have discussed in the main text, as long as $\delta J(y)$ does not vary rapidly on the lattice scale, the effective Dirac theory Eq. (B1) should be a good approximation, and the spatially varying twist-induced gauge field $\tilde{\mathscr{B}}_{z}$ should lead to magnon DLLs in the vicinity of magnon Dirac cones.

We now derive the dispersion of the twist-induced magnon LLs. In the framework of band theory, the effect of the twistinduced vector potential is to shift the magnon Dirac cones at $K$ and $K^{\prime}$. For a specific momentum that is $q_{x}$ on the right of the Dirac point $K$, the Dirac cone is relocated to this momentum by $\tilde{\mathcal{A}}_{x}=-\frac{\hbar}{e} q_{x}$. Therefore a relation can be constructed between the momentum $q_{x}$ and the spatial coordinate $y$ as

$$
q_{x}=-\frac{2}{3 a}\left\{1-\exp \left[\sqrt{1+\frac{3}{4} \lambda^{2}\left(y^{2}+\frac{a}{2} y\right)}-1\right]\right\} .
$$

For transparency, we introduce the parameter

$$
\zeta_{q_{x}}=1+\ln \left(1+\frac{3}{2} a q_{x}\right)=\sqrt{1+\frac{3}{4} \lambda^{2}\left(y^{2}+\frac{a}{2} y\right)},
$$



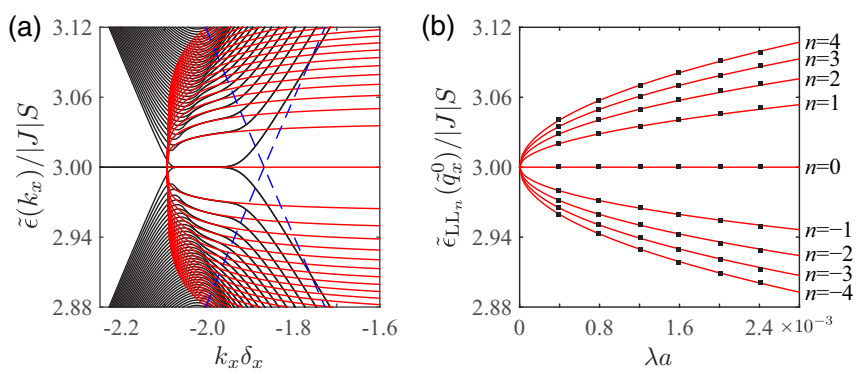

FIG. 7. Dispersive DLLs in a twisted FM nanoribbon. (a) Numerically calculated band structure (black) for a nanoribbon with $2 N=1200 A$ and $B$ sites and $\lambda a=0.0008$. The theoretically predicted LLs (red) from Eq. (B11) are overlaid. The dashed blue curves mark the position of the maximally displaced Dirac cone, which is $\tilde{q}_{x}^{w} \delta_{x}=0.226$ on the right of valley $K$. (b) DLLs as functions of twist at a fixed momentum that is $\tilde{q}_{x}^{0} \delta_{x}=0.115$ on the right of the $K$ point. The theoretically predicted LLs (red) from Eq. (B11) well fit the numerics (black dots) of the first few LLs.

so that Eqs. (B2), (B3), and (B5) may be rewritten as

$$
\begin{gathered}
\tilde{v}_{x}^{\eta}=-\eta \frac{3 J S a}{2 \hbar} e^{1-\zeta_{q_{x}}}, \\
\tilde{v}_{y}^{\eta}=\frac{J S a}{2 \hbar}\left(2+e^{1-\zeta_{q_{x}}}\right), \\
\tilde{\mathcal{B}}_{z}=\eta \frac{\hbar}{2 a^{2} e} \lambda a \sqrt{\frac{4}{3} \frac{\zeta_{q_{x}}^{2}-1}{\zeta_{q_{x}}^{2}}} e^{\zeta_{q_{x}}-1} .
\end{gathered}
$$

Therefore the twist-induced DLLs are characterized by

$$
\begin{aligned}
\tilde{\epsilon}_{\mathrm{LL}_{n}}\left(q_{x}\right)= & -3 J S+\operatorname{sgn}(n) \sqrt{2 n \frac{e \tilde{\mathcal{B}}_{z}}{\hbar} \hbar \tilde{v}_{x}^{\eta} \hbar \tilde{v}_{y}^{\eta}} \\
= & -3 J S-\frac{\sqrt[4]{3}}{\sqrt{2}} J S \sqrt{\lambda a} \sqrt[4]{\zeta_{q_{x}}^{2}-1} \sqrt{\frac{2+e^{1-\zeta_{q_{x}}}}{\zeta_{q_{x}}}} \\
& \times \operatorname{sgn}(n) \sqrt{n},
\end{aligned}
$$

which is reduced to Eq. (19) for a sufficiently small twist $\lambda \rightarrow 0$.

To verify our theory, we perform numerical simulations of a zigzag nanoribbon of a honeycomb ferromagnet with the twist effect incorporated into the lattice Hamiltonian through Eq. (10). For small twists, we find that Eq. (B11) indeed well fits the numerically calculated magnon bands [Fig. 7(a)] between the Dirac cone at the $K$ point and the maximally displaced Dirac cone [dashed blue curves, Fig. 7(a)], which is $\tilde{q}_{x}^{w}=-\frac{2}{3 a}\left\{1-\exp \left[\left(1+\frac{3}{16} \lambda^{2} W^{2}\right)^{1 / 2}-1\right]\right\}$ [see Eq. (B6)] on the right of the $K$ point. We also check the $\sqrt{\lambda a}$ dependence of the first few DLLs. These results are summarized in Fig. 7(b).

For large twists, we find that Eq. (B11) still well matches the numerically calculated LLs in the vicinity of the Dirac point $K$ but begins to deviate from the numerics for large $q_{x}$ with the largest deviation $\sim 8 \%$ occurring at the $\Gamma$ point $\left(q_{x} \delta_{x}=\frac{2 \pi}{3}\right)$ as illustrated in Fig. 8(a). We attribute this deviation to the magnon velocity renormalization in the effective Dirac theory [Eq. (B1)] away from the Dirac points. Nevertheless, we examine the numerically calculated magnon bands connecting the two Dirac cones and find they exhibit $\sqrt{n}$ dependence, thus still being DLLs. Our finding is also (a)

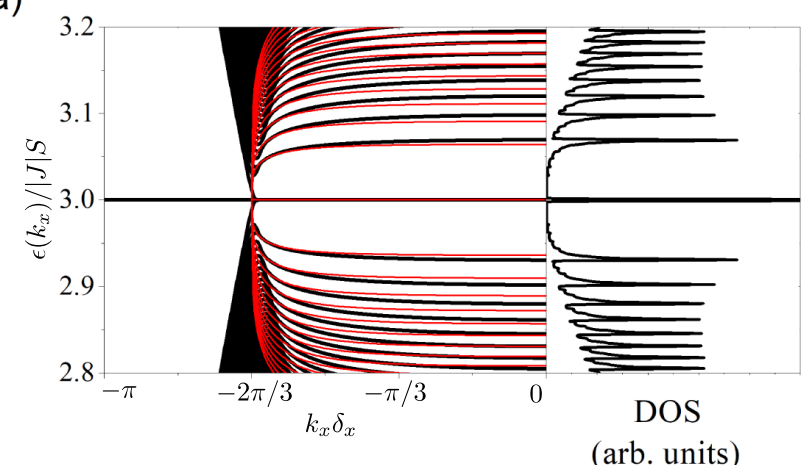

(b)
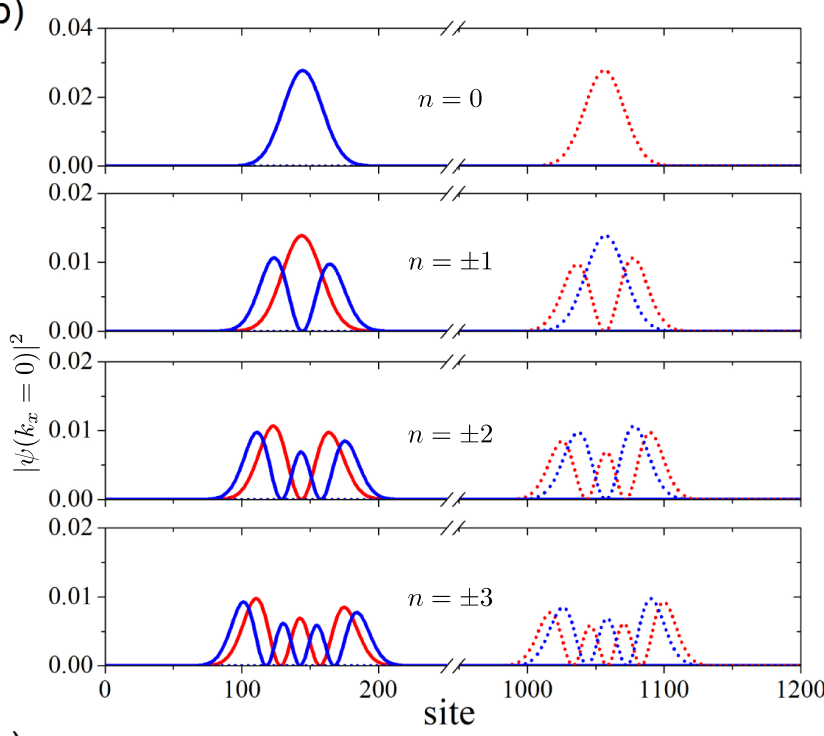

(c)

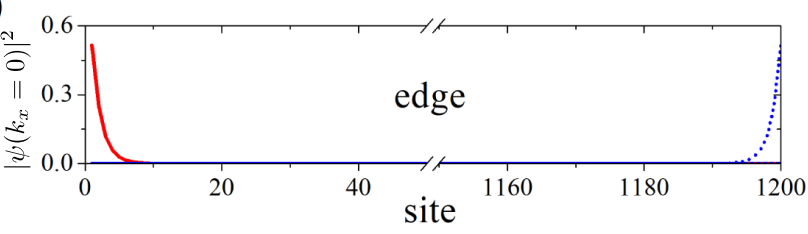

FIG. 8. Dispersive DLLs in a FM nanoribbon with $2 N=1200$ $A$ and $B$ sites in the unit cell, exponentially decaying interactions, and a large twist $\lambda a=0.0023$. (a) Band structure (left panel, black) and DOS (right panel). The DOS is calculated with a small Gaussian broadening $\delta \epsilon /|J| S=5 \times 10^{-4}$. The theoretically predicted LLs (red) from Eq. (B11) are overlaid. (b) DLL wave functions at the $\Gamma$ point, with the LL index shown in the middle of each panel. Red (blue) curves correspond to the $A(B)$ sublattice; solid and dotted curves denote different, degenerate states localized at different positions in the unit cell; and each LL is doubly degenerate. (c) Edge-state wave functions at the $\Gamma$ point. Note that the horizontal scale is different from that in (b).

supported by the calculations of density of states (DOS) [Fig. 8(a), right panel] and the wave functions of the first few LLs at the $\Gamma$ point [Fig. 8(b)]. For completeness, we also present the wave functions of the zigzag edge states as illustrated in Fig. 8(c).

\section{Honeycomb antiferromagnets}

We now consider the Bloch Hamiltonian of antiferromagnets [Eq. (24)] defined in the basis $\left(a_{k}, b_{-k}^{\dagger}\right)^{T}$, with a fictitious 

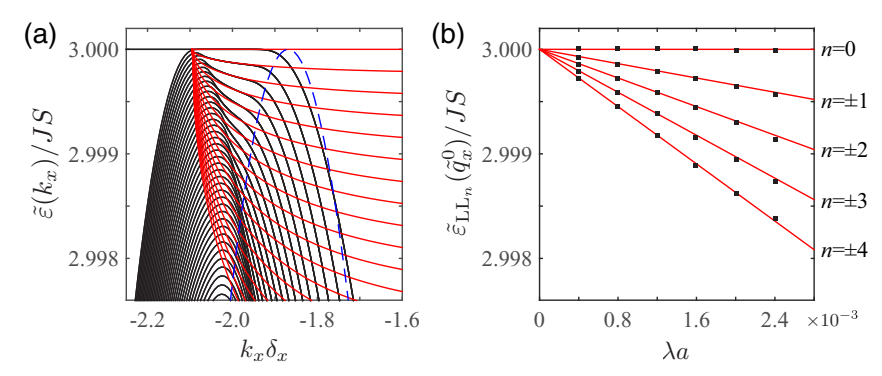

FIG. 9. Dispersive eLLs in a twisted AF nanoribbon. (a) Numerically calculated band structure (black) for a nanoribbon with $2 \mathrm{~N}=$ $1200 A$ and $B$ sites and $\lambda a=0.0008$. The theoretically predicted LLs (red) from Eq. (B14) are overlaid. The dashed blue curves mark the position of the maximally displaced quadratic peak, which is $\tilde{q}_{x}^{w} \delta_{x}=0.226$ on the right of quadratic peak $K$. (b) eLLs as functions of twist at a fixed momentum that is $\tilde{q}_{x}^{0} \delta_{x}=0.115$ on the right of the $K$ point. The theoretically predicted LLs (red) from Eq. (B14) well fit the numerics (black dots) of the first few LLs.

lattice deformation Eq. (15) producing a constant variation $\delta J$ for $J_{1}$ and $J_{2}$. By applying a Bogoliubov transformation, we can obtain the magnon dispersion in the vicinity of the BZ corners,

$$
\begin{aligned}
\tilde{\epsilon}_{q}= & -\frac{1}{6 J S}\left[\hbar \tilde{v}_{x}^{\eta}\left(q_{x}+\eta \frac{2}{3 a} \frac{\delta J}{J+\delta J}\right)\right]^{2} \\
& -\frac{1}{6 J S}\left(\hbar \tilde{v}_{y}^{\eta} q_{y}\right)^{2}+3 J S+2 \delta J S .
\end{aligned}
$$

We can extract from Eq. (B12) the twist-induced potential $\tilde{\mathscr{A}}_{x}=\eta \frac{2 \hbar}{3 e a} \frac{\delta J}{J+\delta J}$, which is exactly the same as that of a ferromagnetic nanoribbon. Therefore, applying the technique detailed in Appendix B 1, we can immediately write down the twist-induced elastic gauge field

$$
\tilde{\mathscr{B}}_{z}=\eta \frac{\hbar}{2 a^{2} e} \lambda a \sqrt{\frac{4}{3} \frac{\zeta_{q_{x}}^{2}-1}{\zeta_{q_{x}}^{2}}} e^{\zeta_{q_{x}}-1}
$$

and the resulting eLLs

$$
\begin{aligned}
\tilde{\varepsilon}_{\mathrm{LL}_{n}}\left(q_{x}\right) & =\sqrt{(3 J S)^{2}-\left|2 n \frac{e}{\hbar} \tilde{\mathscr{B}}_{z} \hbar \tilde{v}_{x}^{\eta} \hbar \tilde{v}_{y}^{\eta}\right|} \\
& =3 J S-\frac{\sqrt{3}}{12} J S \lambda a\left(2+e^{1-\zeta_{q_{x}}}\right) \sqrt{\frac{\zeta_{q_{x}}^{2}-1}{\zeta_{q_{x}}^{2}}}|n|,
\end{aligned}
$$

which is reduced to Eq. (27) for a sufficiently small twist $\lambda \rightarrow 0$.

To test our theory, we perform numerical simulations of a zigzag nanoribbon of a honeycomb antiferromagnet with the twist effect incorporated into the lattice Hamiltonian through Eq. (10). For small twists, we find that Eq. (B14) indeed well captures the momentum dependence of the LLs between the quadratic peak at the $\mathrm{BZ}$ corner $K$ and the maximally displaced quadratic peak [dashed blue curves, Fig. 9(a)], which is $\tilde{q}_{x}^{w}=-\frac{2}{3 a}\left\{1-\exp \left[\left(1+\frac{3}{16} \lambda^{2} W^{2}\right)^{1 / 2}-1\right]\right\}$ on the right of the $K$ point. The twist dependence is also checked and summarized in Fig. 9(b).

When the twist is large, Eq. (B14) can still capture the momentum dependence in the vicinity of $K$ but loses its accuracy

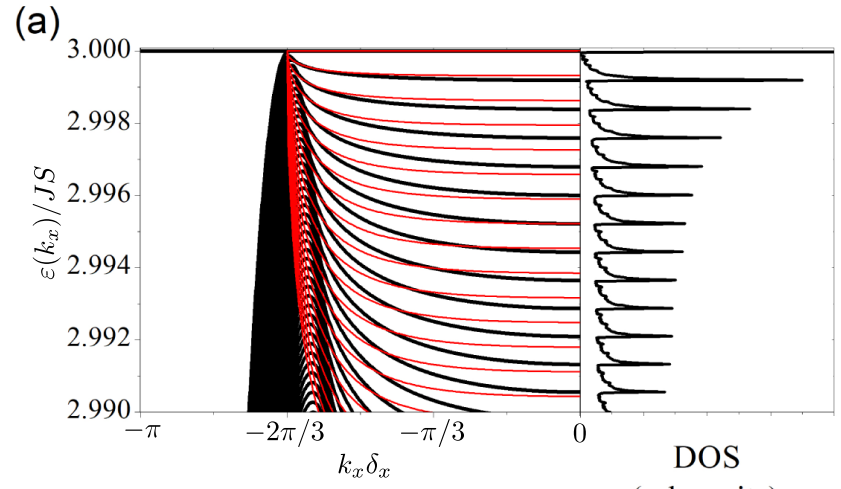

(b)
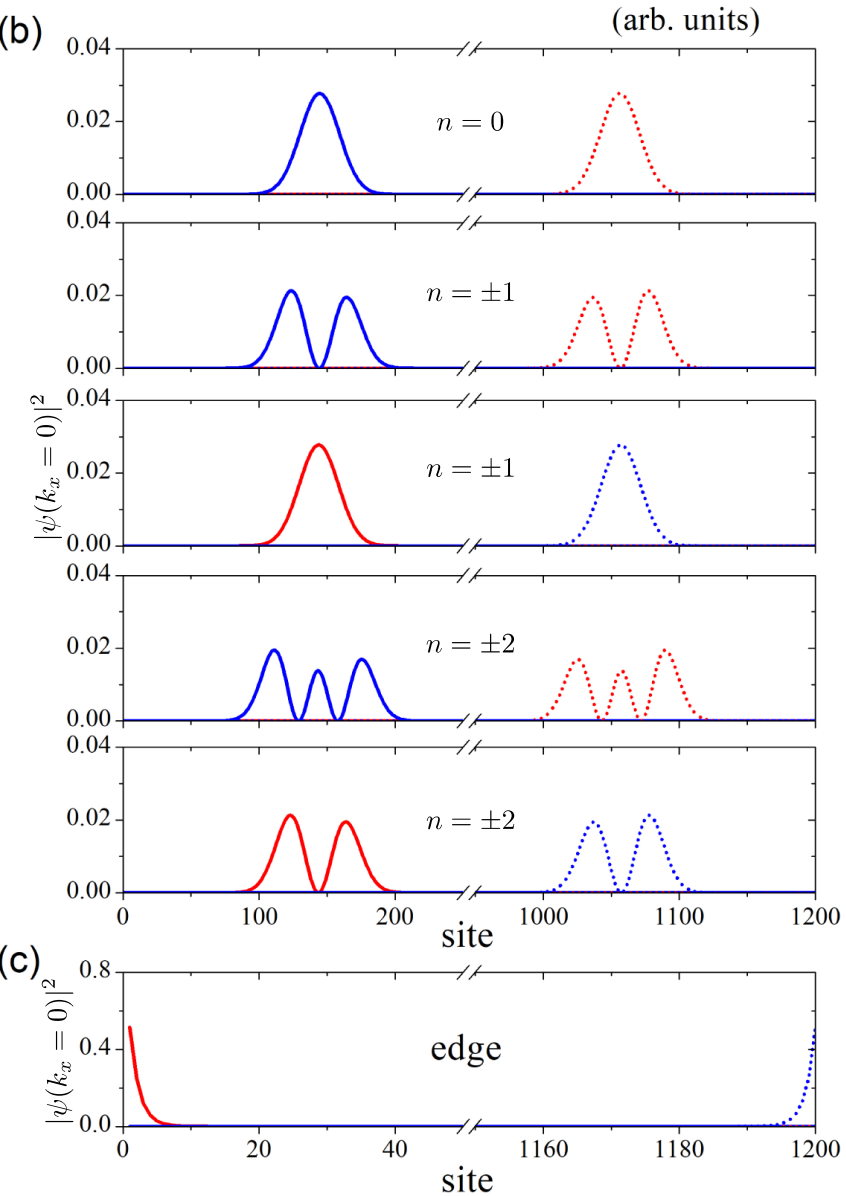

FIG. 10. Dispersive eLLs in an AF nanoribbon with $2 N=1200$ $A$ and $B$ sites in the unit cell, exponentially decaying interactions, and a large twist $\lambda a=0.0023$. (a) Band structure (left panel, black) and DOS (right panel). The DOS is calculated with a small Gaussian broadening $\delta \varepsilon /|J| S=10^{-5}$. The theoretically predicted LLs (red) from Eq. (B14) are overlaid. (b) LL wave functions at the $\Gamma$ point, with the LL index shown in the middle of each panel. Red (blue) curves correspond to the $A(B)$ sublattice; solid and dotted curves denote different, degenerate states localized at different positions in the unit cell; the $n=0$ LL is doubly degenerate, while other LLs are fourfold degenerate. (c) Edge-state wave functions at the $\Gamma$ point. Note that the horizontal scale is different from that in (b).

if too far away from $K$ [Fig. 10(a)]. Again, this is because parameters in the effective theory [Eq. (B12)] are renormalized away from the BZ corners. However, by checking the energies of magnon bands in the vicinity of the $\Gamma$ point, we find these 
bands are still eLLs exhibiting $n$ dependence. Specifically, we numerically calculate at the $\Gamma$ point the DOS [right panel, Fig. 10(a)] and the bulk wave functions [Fig. 10(b)], both of which show features expected from Landau quantization. The wave functions of the zigzag edge states are also presented in Fig. 10(c).

\section{APPENDIX C: SPECTRUM OF HONEYCOMB ANTIFERROMAGNETS}

In Sec. IV, we have derived the magnon Bloch Hamiltonian for honeycomb antiferromagnets [Eq. (24)] defined on a basis containing both magnon creation and annihilation operators, i.e., a magnon particle-hole basis. This implies that the dispersion of the Bloch Hamiltonian cannot be obtained by unitary diagonalization, which generally violates the bosonic statistics of the basis. In this Appendix we will derive the magnon band structure for both the infinite model (Appendix C 1) and the nanoribbon model (Appendix C 2).

\section{Infinite model}

We start from the tight-binding Hamiltonian of an antiferromagnet on the honeycomb lattice [Eq. (23)] and apply Fourier transform of the basis $\left(a_{\boldsymbol{r}}, b_{\boldsymbol{r}}\right)^{T}=$ $N_{\mathrm{uc}}^{-1 / 2} \sum_{k} e^{i k \cdot r}\left(a_{k}, b_{k}\right)^{T}$, where $N_{\mathrm{uc}}$ denotes the number of unit cells. We then obtain

$$
H^{\mathrm{AF}}=\sum_{k}\left(\gamma_{k}^{*} a_{k} b_{-k}+\gamma_{k} a_{k}^{\dagger} b_{-k}^{\dagger}+\gamma_{0} a_{k}^{\dagger} a_{k}+\gamma_{0} b_{k}^{\dagger} b_{k}\right),
$$

where we have used the notations $\gamma_{k}=\sum_{i} J_{i} S e^{i k \cdot \alpha_{i}}$ and $\gamma_{0}=$ $\sum_{i} J_{i} S$ for transparency. To resolve the energy eigenvalues of $H^{\mathrm{AF}}$, we apply Bogoliubov transformation of the basis through

$$
\left(\begin{array}{c}
a_{k} \\
b_{-k}^{\dagger}
\end{array}\right)=\left(\begin{array}{cc}
\cosh \theta_{k} & e^{i \phi_{k}} \sinh \theta_{k} \\
e^{-i \phi_{k}} \sinh \theta_{k} & \cosh \theta_{k}
\end{array}\right)\left(\begin{array}{c}
\alpha_{k} \\
\beta_{-k}^{\dagger}
\end{array}\right),
$$

where we set $e^{i \phi_{k}}=\gamma_{k} /\left|\gamma_{k}\right|$. It is easy to check that $\alpha_{k}$ and $\beta_{k}$ have bosonic statistics, thus being magnon annihilation operators. We rewrite $H^{\mathrm{AF}}$ in terms of $\alpha_{k}$ and $\beta_{k}$ as

$$
\begin{aligned}
H^{\mathrm{AF}}= & \sum_{\boldsymbol{k}}\left(\mathscr{H}_{\boldsymbol{k}}^{0}+\mathscr{H}_{\boldsymbol{k}}^{\alpha \alpha} \alpha_{\boldsymbol{k}}^{\dagger} \alpha_{\boldsymbol{k}}+\mathscr{H}_{\boldsymbol{k}}^{\beta \beta} \beta_{-\boldsymbol{k}}^{\dagger} \beta_{-\boldsymbol{k}}\right. \\
& \left.+\mathscr{H}_{\boldsymbol{k}}^{\alpha \beta} \alpha_{\boldsymbol{k}}^{\dagger} \beta_{-\boldsymbol{k}}^{\dagger}+\mathscr{H}_{\boldsymbol{k}}^{\beta \alpha} \beta_{-\boldsymbol{k}} \alpha_{\boldsymbol{k}}\right),
\end{aligned}
$$

where

$$
\begin{aligned}
\mathscr{H}_{k}^{0} & =-\gamma_{0}+\gamma_{0} \cosh 2 \theta_{\boldsymbol{k}}+\left|\gamma_{\boldsymbol{k}}\right| \sinh 2 \theta_{\boldsymbol{k}}, \\
\mathscr{H}_{\boldsymbol{k}}^{\alpha \alpha} & =\mathscr{H}_{\boldsymbol{k}}^{\beta \beta}=\gamma_{0} \cosh 2 \theta_{\boldsymbol{k}}+\left|\gamma_{\boldsymbol{k}}\right| \sinh 2 \theta_{\boldsymbol{k}}, \\
\mathscr{H}_{\boldsymbol{k}}^{\alpha \beta} & =\mathscr{H}_{\boldsymbol{k}}^{\beta \alpha^{\dagger}}=e^{i \phi_{k}}\left[\gamma_{0} \sinh 2 \theta_{\boldsymbol{k}}+\left|\gamma_{\boldsymbol{k}}\right| \cosh 2 \theta_{\boldsymbol{k}}\right] .
\end{aligned}
$$

We choose the parameter $\theta_{\boldsymbol{k}}$ satisfying

$$
\begin{aligned}
\sinh 2 \theta_{k} & =\frac{-\left|\gamma_{k}\right|}{\sqrt{\gamma_{0}^{2}-\left|\gamma_{k}\right|^{2}}}, \\
\cosh 2 \theta_{k} & =\frac{\gamma_{0}}{\sqrt{\gamma_{0}^{2}-\left|\gamma_{k}\right|^{2}}},
\end{aligned}
$$

so that the off-diagonal terms vanish, $\mathscr{H}_{k}^{\alpha \beta}=\mathscr{H}_{k}^{\beta \alpha^{\dagger}}=$ 0 . The magnon dispersion is then given by $\varepsilon_{\boldsymbol{k}}=\mathscr{H}_{\boldsymbol{k}}^{\alpha \alpha}=$ $\mathscr{H}_{\boldsymbol{k}}^{\beta \beta}=\sqrt{\gamma_{0}^{2}-\left|\gamma_{\boldsymbol{k}}\right|^{2}}$, which is doubly degenerate. Explicitly, we have

$$
\varepsilon_{\boldsymbol{k}}=\sqrt{\left(\sum_{i} J_{i} S\right)^{2}-\left|\sum_{i} J_{i} S e^{i k \cdot \alpha_{i}}\right|^{2}} .
$$

For an isotropic model with $J_{i=1,2,3}=J$, this dispersion is reduced to Eq. (25). For the fictitious lattice deformation Eq. (15), we expand Eq. (C6) in the vicinity of the BZ corners $\boldsymbol{k}_{W}^{\eta}=\left(\eta \frac{4 \pi}{3 \sqrt{3} a}, 0\right)$ and obtain Eq. (26). Besides diagonalizing the magnon Hamiltonian, the Bogoliubov transformation Eq. (C2) also produces an extra term $\mathscr{H}_{\boldsymbol{k}}^{0}$ arising from reorganizing the magnon Hamiltonians [Eqs. (C1) and (C3)] in the particle-hole bases $\left(a_{k}, b_{-k}^{\dagger}\right)^{T}$ and $\left(\alpha_{k}, \beta_{-k}^{\dagger}\right)^{T}$, respectively. The effect of $\mathscr{H}_{\boldsymbol{k}}^{0}$ is to alter the Néel state energy by

$$
\begin{aligned}
\delta E_{N} & =\sum_{\boldsymbol{k}} \mathscr{H}_{\boldsymbol{k}}^{0}=-\sum_{\boldsymbol{k}} \gamma_{0}+\sum_{\boldsymbol{k}} \varepsilon_{\boldsymbol{k}} \\
& =-\sum_{\boldsymbol{k}} \gamma_{0}+\sum_{\boldsymbol{k}} \sqrt{\gamma_{0}^{2}-\left|\gamma_{\boldsymbol{k}}\right|^{2}}<0,
\end{aligned}
$$

which leads to a lower ground-state energy $E_{G}^{\mathrm{AF}}=E_{N}+\delta E_{N}$, reflecting the renormalization of the Néel state due to quantum fluctuations. It is also worth noting that the ground state $|G\rangle$ is the vacuum state for the transformed magnons such that $\left\langle G\left|\alpha_{k}^{\dagger} \alpha_{k}\right| G\right\rangle=\left\langle G\left|\beta_{k}^{\dagger} \beta_{k}\right| G\right\rangle=0$, while $\left\langle G\left|a_{k}^{\dagger} a_{k}\right| G\right\rangle$ and $\left\langle G\left|b_{k}^{\dagger} b_{k}\right| G\right\rangle$ are generally not vanishing.

\section{Nanoribbon model}

In Appendix $\mathrm{C} 1$ we have explained that a proper choice of Bogoliubov transformation [Eq. (C2)] can cancel the offdiagonal terms in $H^{\mathrm{AF}}$. This procedure, however, becomes analytically unfeasible for nanoribbons, where there are a large number of degrees of freedom associated with the tightbinding Hamiltonian Eq. (23). In this section we will propose a practical way to obtain the magnon bands for antiferromagnetic nanoribbons.

For a nanoribbon with $M A(B)$ sublattice sites, the magnon Hamiltonian can be generally written as

$$
H^{\mathrm{AF}}=\sum_{k} \Psi_{k}^{\dagger} \mathscr{H}_{k} \Psi_{k}
$$

where $\Psi_{k}=\left(a_{k, 1}, \ldots, a_{k, M}, b_{-k, 1}^{\dagger}, \ldots, b_{-k, M}^{\dagger}\right)^{T}$ is the basis in which the Bloch Hamiltonian $\mathscr{H}_{k}$ is defined, and a constant term due to reordering magnon operators which lowers the Néel state energy has been neglected. We apply a Bogoliubov transformation $\Psi_{k}=P_{k} \Phi_{k}$, where $\Phi_{k}=$ $\left(\alpha_{k, 1}, \ldots, \alpha_{k, M}, \beta_{-k, 1}^{\dagger}, \ldots, \beta_{-k, M}^{\dagger}\right)^{T}$ is the transformed basis, in which $\alpha_{k, i=1, \ldots, M}$ and $\beta_{k, i=1, \ldots, M}$ are new magnon annihilation operators with bosonic statistics. The transformation matrix $P_{k}$ is $2 M \times 2 M$ in the form

$$
P_{k}=\left(\begin{array}{ll}
U_{k} & V_{k} \\
V_{k} & U_{k}
\end{array}\right),
$$

in which some restrictions have to be imposed on $U_{k}$ and $V_{k}$ matrices in order to preserve the bosonic statistics of the basis. 
Explicitly, we need to have

$$
\begin{aligned}
{\left[a_{k, i}, a_{k, j}^{\dagger}\right] } & =\left[U_{k} U_{k}^{\dagger}-V_{k} V_{k}^{\dagger}\right]_{i j}=\delta_{i j}, \\
{\left[a_{k, i}, b_{-k, j}\right] } & =\left[U_{k} V_{k}^{\dagger}-V_{k} U_{k}^{\dagger}\right]_{i j}=0,
\end{aligned}
$$

which suggests that $P_{\boldsymbol{k}}$ is a paraunitary matrix satisfying $P_{k}^{-1}=\tau^{z} P_{k}^{\dagger} \tau^{z}$, where $\tau^{z}$ is the Pauli matrix specifying magnon particles and holes. If the transformation $P_{k}$ diagonalizes the magnon Hamiltonian, i.e., $P_{k}^{\dagger} \mathscr{H}_{k} P_{k}=$ $\operatorname{diag}\left(\Lambda_{k}^{\alpha}, \Lambda_{-k}^{\beta}\right)$ with the positive-definite diagonal matrix $\Lambda_{\boldsymbol{k}}^{\mu}=\operatorname{diag}\left(\varepsilon_{\boldsymbol{k}, 1}^{\mu}, \ldots, \varepsilon_{\boldsymbol{k}, M}^{\mu}\right)$ where $\mu=\alpha$, $\beta$, we then have

$$
P_{k}^{-1}\left(\tau^{z} \mathscr{H}_{k}\right) P_{k}=\operatorname{diag}\left(\Lambda_{k}^{\alpha},-\Lambda_{-k}^{\beta}\right) .
$$

Consequently, the magnon dispersion of $\mathscr{H}_{\boldsymbol{k}}$ can be obtained by diagonalizing the non-Hermitian matrix $\tau^{z} \mathscr{H}_{\boldsymbol{k}}$ for $M$ positive eigenvalues $\varepsilon_{\boldsymbol{k}, 1}^{\alpha}, \ldots, \varepsilon_{\boldsymbol{k}, M}^{\alpha}$, which are inherently the magnon energies at $\boldsymbol{k}$, and $M$ negative eigenvalues $-\varepsilon_{-k, 1}^{\beta}, \ldots,-\varepsilon_{-k, M}^{\beta}$, whose particle-hole partners are the actual magnon energies at $\boldsymbol{k}$.
Alternatively, the magnon Hamiltonian Eq. (C8) can be rewritten as

$$
H^{\mathrm{AF}}=\frac{1}{2} \sum_{k}\left(\begin{array}{ll}
\Psi_{k}^{\dagger} & \tilde{\Psi}_{-k}
\end{array}\right) \mathscr{H}_{k}^{\mathrm{BdG}}\left(\begin{array}{c}
\Psi_{k} \\
\tilde{\Psi}_{-k}^{\dagger}
\end{array}\right)
$$

where $\left(\Psi_{k}, \tilde{\Psi}_{-k}^{\dagger}\right)^{T}$ is the magnon Nambu basis with $\tilde{\Psi}_{-k}^{\dagger}=\left(a_{-k, 1}^{\dagger}, \ldots, a_{-k, M}^{\dagger}, b_{k, 1}, \ldots, b_{k, M}\right)^{T}$ and the magnon Bogoliubov-de Gennes (BdG) Hamiltonian reads

$$
\mathscr{H}_{k}^{\mathrm{BdG}}=\left(\begin{array}{cc}
\mathscr{H}_{\boldsymbol{k}} & \\
& \mathscr{H}_{-k}^{*}
\end{array}\right) .
$$

We make use of Eq. (C11) and obtain

$$
Q_{k}^{-1} \Gamma^{z} \mathscr{H}_{\boldsymbol{k}}^{\mathrm{BdG}} Q_{k}=\operatorname{diag}\left(\Lambda_{\boldsymbol{k}}^{\alpha},-\Lambda_{-\boldsymbol{k}}^{\beta},-\Lambda_{-\boldsymbol{k}}^{\alpha}, \Lambda_{\boldsymbol{k}}^{\beta}\right),
$$

where $Q_{k}=\operatorname{diag}\left(P_{k}, P_{-k}^{*}\right)$ is the Bogoliubov transformation matrix for $\mathscr{H}_{k}^{\mathrm{BdG}}$ and $\Gamma^{z}=\operatorname{diag}\left(\tau^{z},-\tau^{z}\right)$ is the Pauli matrix defined in Nambu space. It is easy to see that the positive eigenvalues of $\mathscr{H}_{\boldsymbol{k}}^{\mathrm{BdG}}$ directly give the magnon dispersion of the Bloch Hamiltonian $\mathscr{H}_{k}$, while the negative eigenvalues result from trivial redundancy, thus being unphysical.
[1] B. Huang, K.-H. Jin, B. Cui, F. Zhai, J. Mei, and F. Liu, Nat. Commun. 8, 15850 (2017).

[2] C. Şahin, G. Vignale, and M. E. Flatté, Phys. Rev. Mater. 3, 014401 (2019).

[3] X. Meng, T. Pandey, J. Jeong, S. Fu, J. Yang, K. Chen, A. Singh, F. He, X. Xu, J. Zhou et al., Phys. Rev. Lett. 122, 155901 (2019).

[4] J. A. Seijas-Bellido, R. Rurali, J. Íñiguez, L. Colombo, and C. Melis, Phys. Rev. Mater. 3, 065401 (2019).

[5] M. E. Cifuentes-Quintal, O. de la Peña-Seaman, R. Heid, R. de Coss, and K.-P. Bohnen, Phys. Rev. B 94, 085401 (2016).

[6] R. Bistritzer and A. H. MacDonald, Proc. Natl. Acad. Sci. USA 108, 12233 (2011).

[7] Y. Cao, V. Fatemi, A. Demir, S. Fang, S. L. Tomarken, J. Y. Luo, J. D. Sanchez-Yamagishi, K. Watanabe, T. Taniguchi, E. Kaxiras et al., Nature 556, 80 (2018).

[8] Y. Cao, V. Fatemi, S. Fang, K. Watanabe, T. Taniguchi, E. Kaxiras, and P. Jarillo-Herrero, Nature 556, 43 (2018).

[9] Z. Zhu, M. Li, and J. Li, Phys. Rev. B 94, 155121 (2016).

[10] D. Shao, J. Ruan, J. Wu, T. Chen, Z. Guo, H. Zhang, J. Sun, L. Sheng, and D. Xing, Phys. Rev. B 96, 075112 (2017).

[11] S. Guan, Z.-M. Yu, Y. Liu, G.-B. Liu, L. Dong, Y. Lu, Y. Yao, and S. A. Yang, Npj Quantum Mater. 2, 23 (2017).

[12] S. Owerre, J. Phys.: Condens. Matter 30, 245803 (2018).

[13] W. Zhang, K. Luo, Z. Chen, Z. Zhu, R. Yu, C. Fang, and H. Weng, Npj Comput. Mater. 5, 105 (2019).

[14] J. Mutch, W.-C. Chen, P. Went, T. Qian, I. Z. Wilson, A. Andreev, C.-C. Chen, and J.-H. Chu, Sci. Adv. 5, eaav9771 (2019).

[15] F. Guinea, M. Katsnelson, and A. Geim, Nat. Phys. 6, 30 (2010).

[16] N. Levy, S. Burke, K. Meaker, M. Panlasigui, A. Zettl, F. Guinea, A. C. Neto, and M. Crommie, Science 329, 544 (2010).

[17] M. A. Vozmediano, M. Katsnelson, and F. Guinea, Phys. Rep. 496, 109 (2010).
[18] M. C. Rechtsman, J. M. Zeuner, A. Tünnermann, S. Nolte, M. Segev, and A. Szameit, Nat. Photonics 7, 153 (2013).

[19] A. Cortijo, Y. Ferreirós, K. Landsteiner, and M. A. H. Vozmediano, Phys. Rev. Lett. 115, 177202 (2015).

[20] D. I. Pikulin, A. Chen, and M. Franz, Phys. Rev. X 6, 041021 (2016).

[21] A. G. Grushin, J. W. F. Venderbos, A. Vishwanath, and R. Ilan, Phys. Rev. X 6, 041046 (2016).

[22] A. Cortijo, D. Kharzeev, K. Landsteiner, and M. A. H. Vozmediano, Phys. Rev. B 94, 241405(R) (2016).

[23] H. Sumiyoshi and S. Fujimoto, Phys. Rev. Lett. 116, 166601 (2016).

[24] V. Arjona, E. V. Castro, and M. A. H. Vozmediano, Phys. Rev. B 96, 081110(R) (2017).

[25] T. Liu, D. I. Pikulin, and M. Franz, Phys. Rev. B 95, 041201(R) (2017).

[26] T. Liu, M. Franz, and S. Fujimoto, Phys. Rev. B 96, 224518 (2017).

[27] G. Massarelli, G. Wachtel, J. Y. T. Wei, and A. Paramekanti, Phys. Rev. B 96, 224516 (2017).

[28] T. Matsushita, T. Liu, T. Mizushima, and S. Fujimoto, Phys. Rev. B 97, 134519 (2018).

[29] T. Kobayashi, T. Matsushita, T. Mizushima, A. Tsuruta, and S. Fujimoto, Phys. Rev. Lett. 121, 207002 (2018).

[30] E. M. Nica and M. Franz, Phys. Rev. B 97, 024520 (2018).

[31] Y. Ferreiros and M. A. H. Vozmediano, Phys. Rev. B 97, 054404 (2018).

[32] T. Liu and Z. Shi, Phys. Rev. B 99, 214413 (2019).

[33] T. Liu, Phys. Rev. B 102, 045151 (2020).

[34] F. Guinea, A. K. Geim, M. I. Katsnelson, and K. S. Novoselov, Phys. Rev. B 81, 035408 (2010).

[35] Y. Chang, T. Albash, and S. Haas, Phys. Rev. B 86, 125402 (2012). 
[36] S. G. Stuij, P. H. Jacobse, V. Juričić, and C. M. Smith, Phys. Rev. B 92, 075424 (2015).

[37] D.-B. Zhang, G. Seifert, and K. Chang, Phys. Rev. Lett. 112, 096805 (2014).

[38] É. Lantagne-Hurtubise, X.-X. Zhang, and M. Franz, Phys. Rev. B 101, 085423 (2020).

[39] M. Oliva-Leyva, J. E. Barrios-Vargas, and G. G. de la Cruz, Phys. Rev. B 102, 035447 (2020).

[40] Z. Shi and T. Liu, arXiv:2006.09048.

[41] The modulation of wave-function overlap due to the lattice deformation also alters the underlying electronic states and may produce novel magnetic orders, e.g., one that is neither purely ferromagnetic nor purely antiferromagnetic [55] if the electronic bands without the lattice deformation are Dirac-like. However, for magnetic insulators, the inherent ferromagnetism and antiferromagnetism are unlikely to be affected by the lattice deformation if it does not close the electronic band gap.

[42] M. M. Nayga, S. Rachel, and M. Vojta, Phys. Rev. Lett. 123, 207204 (2019).

[43] T. Holstein and H. Primakoff, Phys. Rev. 58, 1098 (1940).

[44] Y. Aharonov and A. Casher, Phys. Rev. Lett. 53, 319 (1984).
[45] B. Huang, G. Clark, E. Navarro-Moratalla, D. R. Klein, R. Cheng, K. L. Seyler, D. Zhong, E. Schmidgall, M. A. McGuire, D. H. Cobden et al., Nature 546, 270 (2017).

[46] S. S. Pershoguba, S. Banerjee, J. C. Lashley, J. Park, H. Ågren, G. Aeppli, and A. V. Balatsky, Phys. Rev. X 8, 011010 (2018).

[47] Y. Shiomi, R. Takashima, and E. Saitoh, Phys. Rev. B 96, 134425 (2017).

[48] Y. Martin and H. K. Wickramasinghe, Appl. Phys. Lett. 50, 1455 (1987).

[49] B. Brockhouse, Phys. Rev. 106, 859 (1957).

[50] X. Wen, C. Qiu, Y. Qi, L. Ye, M. Ke, F. Zhang, and Z. Liu, Nat. Phys. 15, 352 (2019).

[51] C. Brendel, V. Peano, O. J. Painter, and F. Marquardt, Proc. Natl. Acad. Sci. USA 114, E3390 (2017).

[52] B. Perreault, S. Rachel, F. J. Burnell, and J. Knolle, Phys. Rev. B 95, 184429 (2017).

[53] S. Rachel, L. Fritz, and M. Vojta, Phys. Rev. Lett. 116, 167201 (2016).

[54] Y.-H. Ho, E. V. Castro, and M. A. Cazalilla, Phys. Rev. B 96, 155446 (2017).

[55] B. Roy, F. F. Assaad, and I. F. Herbut, Phys. Rev. X 4, 021042 (2014). 